\title{
SPECIES COMPOSITION AND STRUCTURE OF THYSANOPTERA COMMUNITIES IN DIFFERENT MICROHABITATS AT THE PARQUE ESTADUAL DE ITAPUÃ, VIAMÃO, RS*
}

\author{
PINENT, S. M. J. ${ }^{1}$, ROMANOWSKI, H. P. ${ }^{1}$, REDAELLI, L. R. ${ }^{1,2}$ and CAVALLERI, A. ${ }^{1}$ \\ ${ }^{1}$ Programa de Pós-Graduação em Biologia Animal, Instituto de Biociências, Universidade Federal do Rio Grande do Sul, \\ Av. Bento Gonçalves, 9500, Bloco IV, Prédio 43435, CEP 91501-970, Porto Alegre, RS, Brasil \\ ${ }^{2}$ Departamento de Fitossanidade, Faculdade de Agronomia, Universidade Federal do Rio Grande do Sul, \\ Av. Bento Gonçalves, 7712, CEP 91540-000, Porto Alegre, RS, Brasil \\ Correspondence to: Silvia M. J. Pinent, Av. Diário de Notícias, 1625, ap. 1603 T1, \\ Bairro Cristal, CEP 90810-080, Porto Alegre, RS, Brazil, e-mail: silviapi@ portoweb.com.br \\ Received July 5, 2004 - Accepted October 4, 2004 - Distributed August 31, 2006
}

(With 6 figures)

\begin{abstract}
Although thrips are known as inhabitants of flowers, they are also abundant and diverse in other microhabitats. There is an information gap concerning them, especially related to the native fauna in southern Brazil. The structure and composition of the thysanopteran community in different microhabitats was studied at the "Parque Estadual de Itapuã" (30²2' S 51 02' W), RS, southern Brazil. Between June 1999 and May 2001, branches $(n=1,274)$, flowers $(n=774)$, grass tussocks $(n=596)$ and leaf litter $(n=603)$ were sampled systematically in 20 points of four trails (T1 - Pedreira beach, T2 - Araçá beach, T3 - Lagoinha, and T4 - Grota hill). We found 2,197 adult thrips determined in 73 species in 41 genera, of which 37 could be nominated. Four families are represented, Thripidae, Phlaeothripidae, Heterothripidae and Merothripidae, with the first the most abundant $(\mathrm{N}=1,599)$ and with the highest species richness $(\mathrm{S}=32)$. The highest thrips abundance occurred in flowers $\mathrm{N}=1,224$ and the highest number of exclusive species occurred in the leaf litter (27). Frankliniella rodeos Moulton, 1933, Frankliniella gemina Bagnall, 1919 and Smicrothrips particula Hood, 1952 comprise 49.4\% of the total sampled. Regarding T2, we obtained the highest abundance $(\mathrm{N}=935)$ and highest species richness $(\mathrm{S}=43)$. The composition of the faunas in each kind of environment proved very particular.
\end{abstract}

Keywords: thrips, Thysanoptera, communities, microhabitats.

\section{RESUMO}

\section{Estrutura e composição de espécies de comunidades de Thysanoptera em diferentes micro-hábitats no Parque Estadual de Itapuã, Viamão, RS}

Embora os tripes sejam conhecidos como habitantes de flores, também são abundantes e diversos em outros micro-hábitats. Há uma lacuna de informações neste sentido, especialmente sobre a fauna nativa no Sul do Brasil. Entre junho de 1999 a maio de 2001, ramos $(\mathrm{n}=1.274)$, flores $(\mathrm{n}=774)$, touceiras de gramíneas $(\mathrm{n}=596)$ e folhedo $(\mathrm{n}=603)$ foram amostrados sistematicamente em 20 pontos de quatro trilhas (T1 - Praia da Pedreira, T2 - Praia do Araçá, T3 - Lagoinha, e T4 - Morro da Grota). Resultaram 2.197 tripes adultos determinados em 73 espécies de 41 gêneros, das quais 37 puderam ser nominadas. Quatro famílias estão representadas, Thripidae, Phlaeothripidae, Heterothripidae e Merothripidae, sendo a primeira mais abundante $(\mathrm{N}=1.599)$ com a maior riqueza de espécies $(\mathrm{S}=32)$. A maior abundância de tripes ocorreu nas flores $(\mathrm{N}=1.224)$ e o maior número de espécies exclusivas ocorreu no folhedo (27).

\footnotetext{
* Contribuição n ${ }^{\circ}$ 451. Departamento de Zoologia - Programa de Pós-Graduação em Biologia Animal, Instituto de Biociências - UFRGS.
} 
Frankliniella rodeos Moulton, 1933, Frankliniella gemina Bagnall, 1919 and Smicrothrips particula Hood, 1952 compõem $49,4 \%$ do total da amostra. $\mathrm{Na}$ T2 foi obtida maior abundância $(\mathrm{N}=935)$ e maior riqueza de espécies $(\mathrm{S}=43)$. A composição das faunas de cada tipo de ambiente revelou-se muito particular.

Palavras-chave: tripes, Thysanoptera, comunidades, micro-hábitats.

\section{INTRODUCTION}

Thrips are phytophagous insects, sap suckers, but can also work as pollinators, predators and decomposers, some of which feed on aquatic plants (Mound, 2000). Their small size has protected them from attention and thus few people know about them (Kirk, 1996). However, they are both abundant and widely dispersed throughout the world.

About 5,500 species have been described. Mound \& Marullo (1996) listed about 300 thrips species from Mexico, 300 from Costa Rica and 400 from Brazil. Mound (2002) increases that value to more than 700 species. Although Brazil is part of a megadiverse region (Lewinsohn et al., 2001), there is a lack in terms of papers on identifying thrips species and their association with plant species, whether spontaneously growing or cultivated, native or exotic.

Moulton (1948), Hood (1952), De Santis et al. (1980), Monteiro et al. (1999) and Ripa et al. (2001) contributed to the knowledge of thysanopterans of the Neotropical region with important studies of taxonomic nature. However, generally, they deal with species considered as pests. In spite of this fact, there is no information concerning natural communities of thrips in southern Brazil. In a critical analysis on hysanopteran diversity in the Neotropics, Mound (2002) confirms this reality.

Given the constant destruction of natural environments, any information on species diversity has become critically important to the understanding of the tropical communities and their conservation (DeVries et al., 1997).

Of the published Thysanoptera species lists from different parts of the world, few mention the number and distribution of the individuals in their habitats. For example, Von Öettingen (1954 apud Lewis, 1973) listed the species of thrips characteristic of dunes, meadows and forests in Sweden, but these surveys do not analyse community diversity and their spatial patterns.
Considering a wider point of view, surveys of phytophagous insects on host plants contribute directly to the knowledge of an important and diversified segment of local biotas. This information can be analysed in many ways at many scales, per individual plant, per plant population, per habitat and per region, making a diverse array of types of estimates of species richness possible, even from an admittedly incomplete species list (Lewinsohn et al., 2001; Pinent et al., 2003).

The aim of this paper is to describe the structure and composition of thrips species living in different microhabitats at the "Parque Estadual de Itapuã", Viamão, RS.

\section{MATERIALS AND METHODS}

\section{Study area}

This research was done in the "Parque Estadual de Itapuã” (PEI) (30 22' S 51 ${ }^{\circ} 02^{\prime} \mathrm{W}$ ), Viamão, RS, 57 km from Porto Alegre. The Park has an area of 5,566.50 ha including coastal restinga vegetation, as well as granitic hills. The plant cover is varied, including forests and fields, with a great diversity of physiognomic-floristic types (Rio Grande do Sul. Secretaria da Agricultura e Abastecimento, 1997) and was fully described by Pinent et al. (2003).

\section{Sampling}

Samplings were taken from June 1999 to May 2001, in intervals from 30 to 45 days. Four preexisting trails, distinct in terms of their vegetation and level of anthropic impact, were selected: $\mathrm{T} 1$ - Pedreira beach trail - extends from the beach, next to the foot of the Fortaleza hill, relatively less impact, dense subtropical rainforest; T2 - Araçá beach trail - extends from the road which has access to the beach towards Araçá beach. It is steep and quite altered from the past as today it is in different stages of recuperation. It has rupestral vegetation, bushy secondary vegetation and low 
forest; T3 - Lagoinha trail - it is parallel to the beach, between dunes and a swampy area known as Lagoinha; fields, xerophytic vegetation and restinga forest with a strong presence of fig trees and T4 - Grota Hill trail - halfway to the top of the Grota hill with a granitic geologic formation. It has rupestral vegetation, bushy secondary vegetation and fields with scattered butia palm trees.

In each of the four trails, five points were tagged along $500 \mathrm{~m}$, to the right and to the left, alternately, evenly spaced at every $100 \mathrm{~m}$ and, at each point, two sites - one immediately at the edge of the trail and another $1.5 \mathrm{~m}$ perpendicularly far from it. At each one of the sites, samples of the four microhabitats were taken, namely flowers, branches, grass tussocks and leaf litter, defined as sheltering different species of Thysanoptera according to Mound \& Kibby (1998) and Shuji Okajima (pers. comm.).

Three flowers per site were sampled (when present). Branches with a maximum length of $20 \mathrm{~cm}$ were sampled from two plants, a priori different. From each of these plants, shoots were taken from three equidistant strata on the plant, up to a maximum height of $1.80 \mathrm{~m}$ from the soil. One grass tussock per site was sampled. To avoid the thrips escaping, the plant parts were first put in a plastic bag and cut with scissors close to the ground. The leaf litter was sampled manually so that all its vertical strata were represented. The portion of leaf litter taken was placed in a standard container so as to sample a volume of approximately $250 \mathrm{~cm}^{3}$, with samples then transferred to plastic bags.

\section{Sorting}

In the laboratory, we separated thrips from all of the sampling units with the help of a fine, soft hair brush under a stereoscopic microscope. All thrips were then placed in AGA preserving solution (60\% alcohol; glacial acetic acid; glycerine at proportions of 10:1:1).

To extract the thrips from the leaf litter, the units were kept for a period of $72 \mathrm{~h}$ in a Berlese funnel and the individuals then extracted were also transferred to AGA.

The adults were mounted on microscopy slides based on the methodology proposed by Palmer et al. (1989) and Mound \& Kibby (1998) and identified with the help of taxonomic keys in Mound \& Marullo (1996), Sakimura \& O’Neill
(1979), Moritz et al., (2001), Monteiro et al. (2001), among others.

Aiming to register the diversity of plants/ thrips associations, in the cases when it was not possible to determine a species from the available keys, individuals were identified by subfamily and/ or genus and discriminated by a numeric code.

The thrips identified were deposited in the collection of the "Laboratório de Ecologia de Insetos" of the "Departamento de Zoologia" of "Instituto de Biociências" at the "Universidade Federal do Rio Grande do Sul".

Plants were herborized, kept as exsiccates and identified according to Brack et al. (1998), Lorenzi \& Moreira (1999) and from consultation with the herbarium of the "Departamento de Botânica" also at the "Universidade Federal do Rio Grande do Sul". The classification system adopted was that of Cronquist (1988).

The cumulative species curves were adjusted using EstimateS software (Colwell, 2000).

\section{RESULTS AND DISCUSSION}

A total of 19 sampling occasions resulted in 2,197 adult individuals collected. Of the 73 species, 2 were identified from the subfamily level, 34 from genus and 37 are nominated (Table 1).

Four families are represented, of which Thripidae had the highest abundance, with 1,599 individuals in 32 species. Of the nine Thysanoptera families, Thripidae is a large and diverse family worldwide, with 1,700 species according to Mound \& Marullo (1996). It is distributed worldwide, and encompasses most of the species which are considered pests (Kirk, 1996). Monteiro et al. (1996) registered 23 species in this family in São Paulo State, among them Microcephalothrips abdominalis Cawford, 1910, also found in this work.

For Phlaeothripidae we registered 559 individuals, represented by 35 species. This family is widely distributed in the world, but with a higher representation in tropical regions, as pointed out by Kirk (1996), who cites a total of 39 species for Great Britain. Indeed, from the species found in this study, only 13 could be identified at the level of species. Mound \& Marullo (1996) interpret this family as a confused assemblage of approximately 
TABLE 1

Species of thrips, total number of individuals $(\mathrm{N})$, simple (F) and relative cumulative frequency (F), number of individuals per sampling trail (T1 - Pedreira beach, T2 - Araçá beach, T3 - Lagoinha, T4 - Grota hill) and by microhabitat (F - flowers, B - branches, G - grass tussocks, L - leaf litter) in the Parque Estadual de Itapuã (30 $\left.{ }^{\circ} 22^{\prime} \mathrm{S} 51^{\circ} \mathrm{02}^{\prime} \mathrm{W}\right)$, Viamão, RS. June 1999 to May 2001.

\begin{tabular}{|c|c|c|c|c|c|c|c|c|c|c|c|c|}
\hline \multirow{2}{*}{\multicolumn{2}{|c|}{ Thrips species }} & $\mathbf{N}$ & $F$ & $\mathbf{F}$ & \multicolumn{4}{|c|}{ Trails } & \multicolumn{4}{|c|}{ Microhabitats } \\
\hline & & & $(\%)$ & $(\%)$ & T1 & T2 & T3 & T4 & $\mathbf{F}$ & B & G & $\mathbf{L}$ \\
\hline & \begin{tabular}{|l|} 
Frankliniella rodeos \\
Moulton, 1933 \\
(Thripidae)
\end{tabular} & 478 & 21,76 & 21,76 & 44 & 247 & 34 & 153 & 460 & 16 & & 2 \\
\hline & $\begin{array}{l}\text { Frankliniella gemina } \\
\text { Bagnall, } 1919 \\
\text { (Thripidae) }\end{array}$ & 381 & 17,34 & 39,10 & 112 & 218 & 2 & 49 & 347 & 33 & & 1 \\
\hline & $\begin{array}{l}\text { Smicrothrips particula } \\
\text { Hood, } 1952 \\
\text { (Phlaeothripidae) }\end{array}$ & 226 & 10,29 & 49,39 & 155 & 46 & 7 & 18 & 7 & 12 & 207 & \\
\hline & \begin{tabular}{|l|} 
Paraleucothrips minusculus \\
(Johansen, 1983) \\
(Thripidae)
\end{tabular} & 193 & 8,78 & 58,17 & 40 & 90 & 41 & 22 & 45 & 145 & & 3 \\
\hline & $\begin{array}{l}\text { Neohydatothrips flavens } \\
\text { (Moulton, 1941) } \\
\text { (Thripidae) }\end{array}$ & 142 & 6,46 & 64,63 & 10 & 34 & 36 & 62 & 13 & 116 & & 13 \\
\hline • & $\begin{array}{l}\text { Frankliniella insularis } \\
\text { (Franklin, 1908) } \\
\text { (Thripidae) }\end{array}$ & 106 & 4,82 & 69,46 & & 103 & & 3 & 106 & & & \\
\hline & $\begin{array}{l}\text { Paraleucothrips sp. } \\
\text { (Thripidae) }\end{array}$ & 83 & 3,78 & 73,24 & 1 & 41 & 23 & 18 & 28 & 55 & & \\
\hline & $\begin{array}{l}\text { Craniothrips urichi } \\
\text { Bagnall, 1915 } \\
\text { (Phlaeothripidae) }\end{array}$ & 71 & 3,23 & 76,47 & 55 & 16 & & & 11 & 60 & & \\
\hline & $\begin{array}{l}\text { Haplothrips sp. } \\
\text { (Phlaeothripidae) }\end{array}$ & 65 & 2,96 & 79,43 & 42 & 23 & & & 7 & 58 & & \\
\hline & $\begin{array}{l}\text { Frankliniella bertelsi } \\
\text { (De Santis, 1967) } \\
\text { (Thripidae) }\end{array}$ & 56 & 2,55 & 81,98 & & 11 & & 45 & 44 & 12 & & \\
\hline & $\begin{array}{l}\text { Haplothrips fiebrigi } \\
\text { Priesner, } 1931 \\
\text { (Phlaeothripidae) }\end{array}$ & 32 & 1,46 & 83,43 & & 2 & 3 & 27 & 30 & 2 & & \\
\hline - & $\begin{array}{l}\text { Heterothrips sp.1 } \\
\text { (Heterothripidae) }\end{array}$ & 30 & 1,37 & 84,80 & & & 10 & 20 & 30 & & & \\
\hline & $\begin{array}{l}\text { Adraneothrips alternatus } \\
\text { Hood, } 1925 \\
\text { (Phlaeothripidae) }\end{array}$ & 26 & 1,18 & 85,98 & 8 & 5 & 12 & 1 & 1 & 1 & & 24 \\
\hline$*$ & $\begin{array}{l}\text { Glyptothrips sp. } \\
\text { (Phlaeothripidae) }\end{array}$ & 22 & 1,00 & 86,98 & 21 & & & 1 & & & & 22 \\
\hline - & $\begin{array}{l}\text { Heliothrips haemorrhoidalis } \\
\text { (Bouché, 1833) } \\
\text { (Thripidae) }\end{array}$ & 21 & 0,96 & 87,94 & 5 & 9 & 6 & 1 & & 21 & & \\
\hline - & $\begin{array}{l}\text { Frankliniella } \text { sp. } \\
\text { (Thripidae) }\end{array}$ & 20 & 0,91 & 88,85 & & 13 & & 7 & 20 & & & \\
\hline$\Delta$ & \begin{tabular}{|l|} 
Bregmatothrips venustus \\
(Hood, 1912) \\
(Thripidae)
\end{tabular} & 15 & 0,68 & 89,53 & 1 & 1 & 13 & & & & 15 & \\
\hline
\end{tabular}


TABLE 1

Continued..

\begin{tabular}{|c|c|c|c|c|c|c|c|c|c|c|c|c|}
\hline \multirow{2}{*}{\multicolumn{2}{|c|}{ Thrips species }} & \multirow{3}{*}{$\begin{array}{l}\mathbf{N} \\
15\end{array}$} & \multirow{2}{*}{\begin{tabular}{|l|}
$F$ \\
$(\%)$
\end{tabular}} & \multirow{2}{*}{\begin{tabular}{|c|}
$\mathbf{F}$ \\
$(\%)$ \\
\end{tabular}} & \multicolumn{4}{|c|}{ Trails } & \multicolumn{4}{|c|}{ Microhabitats } \\
\hline & & & & & T1 & T2 & T3 & T4 & $\mathbf{F}$ & B & G & $\mathbf{L}$ \\
\hline - & \begin{tabular}{|l|} 
Frankliniella serrata \\
Moulton, 1933 \\
(Thripidae) \\
\end{tabular} & & 0,68 & 90,21 & & 3 & & 12 & 15 & & & \\
\hline$*$ & $\begin{array}{l}\text { Adraneothrips fuscicollis } \\
\text { Hood, 1925 } \\
\text { (Phlaeothripidae) }\end{array}$ & 13 & 0,59 & 90,81 & 6 & & 5 & 2 & & & & 13 \\
\hline$*$ & $\begin{array}{l}\text { Allothrips? brasilianus } \\
\text { Hood, } 1955 \\
\text { (Phlaeothripidae) }\end{array}$ & 12 & 0,55 & 91,35 & & & 10 & 2 & & & & 12 \\
\hline & $\begin{array}{l}\text { Ceratothripoides } \\
\text { lagoenacollus } \\
\text { (Moulton, 1933) } \\
\text { (Thripidae) } \\
\end{array}$ & 12 & 0,55 & 91,90 & & 10 & & 2 & 11 & 1 & & \\
\hline • & $\begin{array}{l}\text { Microcephalothrips } \\
\text { abdominalis } \\
\text { (Crawford, 1910) } \\
\text { (Thripidae) } \\
\end{array}$ & 11 & 0,50 & 92,40 & & 2 & 8 & 1 & 11 & & & \\
\hline & $\begin{array}{l}\text { Liothrips sp.1 } \\
\text { (Phlaeothripidae) }\end{array}$ & 9 & 0,41 & 92,81 & 1 & 3 & 5 & & 4 & 3 & 2 & \\
\hline * & $\begin{array}{l}\text { Adraneothrips sp. } \\
\text { (Phlaeothripidae) }\end{array}$ & 8 & 0,36 & 93,17 & 1 & 3 & 3 & 1 & & & & 8 \\
\hline$\div$ & $\begin{array}{l}\text { Eurythrips tarsalis } \\
\text { Hood, } 1925 \\
\text { (Phlaeothripidae) }\end{array}$ & 8 & 0,36 & 93,54 & & & & 8 & & & & 8 \\
\hline - & $\begin{array}{l}\text { Frankliniella nakaharai } \\
\text { Sakimura \& O'Neill, } 1979 \\
\text { (Thripidae) }\end{array}$ & 8 & 0,36 & 93,90 & & 8 & & & & 8 & & \\
\hline • & $\begin{array}{l}\text { Frankliniella williamsi } \\
\text { Hood, } 1915 \\
\text { (Thripidae) }\end{array}$ & 8 & 0,36 & 94,26 & & 3 & & 5 & 8 & & & \\
\hline$*$ & $\begin{array}{l}\text { Stephanothrips sp. } \\
\text { (Phlaeothripidae) }\end{array}$ & 8 & 0,36 & 94,63 & & 1 & 5 & 2 & & & & 8 \\
\hline - & $\begin{array}{l}\text { Heterothrips marginatus } \\
\text { Hood, } 1954 \\
\text { (Heterothripidae) }\end{array}$ & 7 & 0,32 & 94,95 & & 7 & & & 7 & & & \\
\hline$\Delta$ & $\begin{array}{l}\text { Plesiothrips sp. } 1 \\
\text { (Thripidae) }\end{array}$ & 6 & 0,27 & 95,49 & 5 & & 1 & & & & 6 & \\
\hline$*$ & $\begin{array}{l}\text { Stephanothrips occidentalis } \\
\text { Hood \& Williams, } 1925 \\
\text { (Phlaeothripidae) }\end{array}$ & 6 & 0,27 & 95,77 & 1 & & 3 & 2 & & & & 6 \\
\hline & $\begin{array}{l}\text { Trichromothrips sp. } \\
\text { (Thripidae) }\end{array}$ & 6 & 0,27 & 95,22 & & & 1 & 5 & 1 & & 5 & \\
\hline$*$ & $\begin{array}{l}\text { Eurythrips subflavus } \\
\text { Hood, } 1950 \\
\text { (Phlaeothripidae) }\end{array}$ & 5 & 0,23 & 95,99 & 4 & & & 1 & & & & 5 \\
\hline & $\begin{array}{l}\text { Hoodothrips lineatus } \\
\text { (Hood, 1927) } \\
\text { (Thripidae) }\end{array}$ & 5 & 0,23 & 96,22 & 4 & 1 & & & 2 & 3 & & \\
\hline & $\begin{array}{l}\text { Karnyothrips sp. } \\
\text { (Phlaeothripidae) }\end{array}$ & 5 & 0,23 & 96,45 & 2 & 2 & 1 & & 2 & 1 & 2 & \\
\hline
\end{tabular}


TABLE 1

Continued...

\begin{tabular}{|c|c|c|c|c|c|c|c|c|c|c|c|c|}
\hline \multirow{2}{*}{\multicolumn{2}{|c|}{ Thrips species }} & $\mathbf{N}$ & $F$ & $\mathbf{F}$ & \multicolumn{4}{|c|}{ Trails } & \multicolumn{4}{|c|}{ Microhabitats } \\
\hline & & & $(\%)$ & $(\%)$ & T1 & T2 & T3 & T4 & $\mathbf{F}$ & B & G & $\mathbf{L}$ \\
\hline & $\begin{array}{l}\text { Merothrips sp. } \\
\text { (Merothripidae) }\end{array}$ & 5 & 0,23 & 96,68 & 1 & 4 & & & & 1 & 4 & \\
\hline - & $\begin{array}{l}\text { Neohydatothrips fasciatus } \\
\text { (Moulton, 1938) } \\
\text { (Thripidae) }\end{array}$ & 5 & 0,23 & 96,90 & & 4 & & 1 & & 5 & & \\
\hline$\Delta$ & $\begin{array}{l}\text { Plesiothrips sp. } 2 \\
\text { (Thripidae) }\end{array}$ & 5 & 0,23 & 97,13 & & & 5 & & & & 5 & \\
\hline+ & \begin{tabular}{|l} 
Eurythrips citricornis \\
(Hood, 1954) \\
(Phlaeothripidae)
\end{tabular} & 4 & 0,18 & 97,31 & & 2 & & 2 & & & & 4 \\
\hline$*$ & \begin{tabular}{|l}
$\begin{array}{l}\text { Holopothrips sp.1 } \\
\text { (Phlaeothripidae) }\end{array}$ \\
\end{tabular} & 4 & 0,18 & 97,50 & 1 & 3 & & & & & & 4 \\
\hline - & \begin{tabular}{|l}
$\begin{array}{l}\text { Thripinae sp. } 1 \\
\text { (Thripidae) }\end{array}$ \\
\end{tabular} & 4 & 0,18 & 97,68 & 2 & 2 & & & 4 & & & \\
\hline - & \begin{tabular}{|l} 
Thripinae sp.2 \\
(Thripidae)
\end{tabular} & 4 & 0,18 & 97,86 & 1 & & & 3 & 4 & & & \\
\hline+ & \begin{tabular}{|l|} 
Eurythrips sp. 1 \\
(Phlaeothripidae)
\end{tabular} & 3 & 0,14 & 98,00 & 1 & 2 & & & & & & 3 \\
\hline - & $\begin{array}{l}\text { Aurantothrips orchidearum } \\
\text { (Bondar, 1931) } \\
\text { (Thripidae) }\end{array}$ & 2 & 0,09 & 98,09 & & & & 2 & & 2 & & \\
\hline+ & $\begin{array}{l}\text { Chamaeothrips jucundus } \\
\text { (Hood, 1954) } \\
\text { (Phlaeothripidae) }\end{array}$ & 2 & 0,09 & 98,18 & & & & 2 & & & & 2 \\
\hline+ & $\begin{array}{l}\text { Eurythrips sp.2 } \\
\text { (Phlaeothripidae) }\end{array}$ & 2 & 0,09 & 98,27 & & 2 & & & & & & 2 \\
\hline & \begin{tabular}{|l|} 
Frankliniella trinidadensis \\
Hood, 1942 \\
(Thripidae)
\end{tabular} & 2 & 0,09 & 98,36 & & & 1 & 1 & & & 1 & 1 \\
\hline - & $\begin{array}{l}\text { Halmathrips sp. } \\
\text { (Thripidae) }\end{array}$ & 2 & 0,09 & 98,45 & & & 2 & & & 2 & & \\
\hline$\cdot$ & $\begin{array}{l}\text { Heterothrips sp.2 } \\
\text { (Heterothripidae) }\end{array}$ & 2 & 0,09 & 98,54 & & 2 & & & & 2 & & \\
\hline$\cdot$ & $\begin{array}{l}\text { Heterothrips sp. } 3 \\
\text { (Heterothripidae) }\end{array}$ & 2 & 0,09 & 98,63 & & 2 & & & 2 & & & \\
\hline - & \begin{tabular}{|l|}
$\begin{array}{l}\text { Holopothrips sp. } 3 \\
\text { (Phlaeothripidae) }\end{array}$ \\
\end{tabular} & 2 & 0,09 & 98,73 & & 2 & & & & 2 & & \\
\hline$\cdot$ & $\begin{array}{l}\begin{array}{l}\text { Kurtomathrips sp. } \\
\text { (Thripidae) }\end{array} \\
\end{array}$ & 2 & 0,09 & 98,82 & & 2 & & & 2 & & & \\
\hline$\cdot$ & $\begin{array}{l}\text { Liothrips sp.2 } \\
\text { (Phlaeothripidae) }\end{array}$ & 2 & 0,09 & 98,91 & & & 2 & & & 2 & & \\
\hline - & \begin{tabular}{|l|} 
Liothrips sp.3 \\
(Phlaeothripidae)
\end{tabular} & 2 & 0,09 & 99,00 & 2 & & & & & 2 & & \\
\hline * & \begin{tabular}{|l|}
$\begin{array}{l}\text { Malacothips sp. } \\
\text { (Phlaeothripidae) }\end{array}$ \\
\end{tabular} & 2 & 0,09 & 99,09 & 2 & & & & & & & 2 \\
\hline+ & $\begin{array}{l}\begin{array}{l}\text { Terthrothrips sp. } 1 \\
\text { (Phlaeothripidae) }\end{array} \\
\end{array}$ & 2 & 0,09 & 99,18 & & 1 & & 1 & & & & 2 \\
\hline+ & $\begin{array}{l}\text { Tylothrips forticauda } \\
\text { (Hood, 1954) } \\
\text { (Phlaeothripidae) } \\
\end{array}$ & 2 & 0,09 & 99,27 & 2 & & & & & & & 2 \\
\hline
\end{tabular}


TABLE 1

Continued...

\begin{tabular}{|c|c|c|c|c|c|c|c|c|c|c|c|c|}
\hline \multirow{2}{*}{\multicolumn{2}{|c|}{ Thrips species }} & \multirow{3}{*}{$\begin{array}{l}\mathbf{N} \\
1\end{array}$} & \multirow{2}{*}{\begin{tabular}{|c|}
$F$ \\
$(\%)$ \\
\end{tabular}} & \multirow{2}{*}{\begin{tabular}{|c|}
$\mathbf{F}$ \\
$(\%)$ \\
\end{tabular}} & \multicolumn{4}{|c|}{ Trails } & \multicolumn{4}{|c|}{ Microhabitats } \\
\hline & & & & & T1 & T2 & T3 & T4 & $\mathbf{F}$ & B & G & $\mathbf{L}$ \\
\hline$*$ & $\begin{array}{l}\text { ?Arpediothrips sp. } \\
\text { (Thripidae) }\end{array}$ & & 0,05 & 99,32 & & & 1 & & & & & 1 \\
\hline$*$ & $\begin{array}{l}\text { ?Preeriella } \text { sp. } \\
\text { (Phlaeothripidae) }\end{array}$ & 1 & 0,05 & 99,36 & & 1 & & & & & & 1 \\
\hline+ & $\begin{array}{l}\text { Arachisothrips millsi } \\
\text { Stannard, } 1952 \\
\text { (Thripidae) }\end{array}$ & 1 & 0,05 & 99,41 & & 1 & & & & & & 1 \\
\hline • & $\begin{array}{l}\text { Chaetisothrips striatus } \\
\text { (Hood, 1935) } \\
\text { (Thripidae) }\end{array}$ & 1 & 0,05 & 99,45 & 1 & & & & 1 & & & \\
\hline$*$ & $\begin{array}{l}\text { Chthonothrips nigrocinctus } \\
\text { (Hood, 1957) } \\
\text { (Phlaeothripidae) }\end{array}$ & 1 & 0,05 & 99,50 & & 1 & & & & & & 1 \\
\hline$\overline{\Delta \Delta}$ & $\begin{array}{l}\text { Coremothrips sp. } \\
\text { (Thripidae) }\end{array}$ & 1 & 0,05 & 99,54 & 1 & & & & & & 1 & \\
\hline$*$ & $\begin{array}{l}\text { Eschatothrips sp. } \\
\text { (Phlaeothripidae) }\end{array}$ & 1 & 0,05 & 99,59 & & & 1 & & & & & 1 \\
\hline * & $\begin{array}{l}\text { Eurythrips sp.3 } \\
\text { (Phlaeothripidae) }\end{array}$ & 1 & 0,05 & 99,64 & & & & 1 & & & & 1 \\
\hline$\cdot$ & $\begin{array}{l}\text { Frankliniella schultzei } \\
\text { (Trybom, 1910) } \\
\text { (Thripidae) }\end{array}$ & 1 & 0,05 & 99,68 & & & 1 & & & 1 & & \\
\hline$*$ & $\begin{array}{l}\text { Haplothrips? gowdeyi } \\
\text { (Franklin, 1908) } \\
\text { (Phlaeothripidae) }\end{array}$ & 1 & 0,05 & 99,73 & & 1 & & & & & & 1 \\
\hline$*$ & $\begin{array}{l}\text { Holopothrips sp.2 } \\
\text { (Phlaeothripidae) }\end{array}$ & 1 & 0,05 & 99,77 & & & & 1 & & & & 1 \\
\hline$\Delta$ & $\begin{array}{l}\text { Leucothrips? nigripennis } \\
\text { Reuter, } 1904 \\
\text { (Thripidae) } \\
\end{array}$ & 1 & 0,05 & 99,82 & 1 & & & & & & 1 & \\
\hline$*$ & $\begin{array}{l}\text { Neosmerinthothrips sp. } \\
\text { (Phlaeothripidae) }\end{array}$ & 1 & 0,05 & 99,86 & & 1 & & & & & & 1 \\
\hline$*$ & $\begin{array}{l}\begin{array}{l}\text { Sophiothrips sp. } \\
\text { (Phlaeothripidae) }\end{array} \\
\end{array}$ & 1 & 0,05 & 99,91 & & & 1 & & & & & 1 \\
\hline+ & $\begin{array}{l}\begin{array}{l}\text { Terthrothrips sp. } 2 \\
\text { (Phlaeothripidae) }\end{array} \\
\end{array}$ & 1 & 0,05 & 99,95 & & & & 1 & & & & 1 \\
\hline$\cdot$ & $\begin{array}{l}\text { Thrips australis } \\
\text { (Bagnall, 1915) } \\
\text { (Thripidae) }\end{array}$ & 1 & 0,05 & 100 & 1 & & & & 1 & & & \\
\hline & Total & 2.197 & 100 & & 534 & 935 & 243 & 485 & 1.224 & 566 & 249 & 158 \\
\hline
\end{tabular}

- exclusive to flowers; - exclusive to shoots; $\Delta$ exclusive to grass tussocks; and exclusive to the leaf litter.

3,000 described species and a great number of species yet to describe.

In the present work we registered 41 individuals in four species of Heterothripidae. The 70 species of this family (Mound \& Kibby, 1998) are mentioned by Del Claro et al. (1997) as inhabitants of flowers and exclusive to the New
World. In Merothripidae five individuals in only one species of the genus Merothrips Hood, 1912 were registered. This family, revised by Mound \& O'Neill (1974), includes 17 species in three genera distributed in the tropics and subtropics, with very small individuals and only rarely found. The finding regarding grasses is worth mentioning. 
The number of new species found in the sampling constantly increased, indicating a great richness of thysanopteran communities in the PEI (Fig. 1). Such a trend is maintained for the four microhabitats (Fig. 2). The observed pattern for tussocks and leaf litter, however, is worth mentioning. In tussocks, even though the number of species has increased, the fauna seems to typically include a reduced number of species. In the leaf litter, the species richness is remarkable: the number is similar and increases with the same pace as the successive samples observed in flowers and branches.

In the overall abundance distribution of thrips species (Fig. 3) three dominant species can be observed (relative frequency above 0,1 ) - F. rodeos Moulton, 1933, Frankliniella gemina Bagnall, 1919 and Smicrothrips particula Hood, 1952, 11 intermediary species (relative frequency between 0.1 and 0.01 ) and 59 rare species (relative frequency lower than 0.01). Sixteen species were represented by only one individual. Out of the 73 identified species, the most common three consist of $49.4 \%$ of the individuals in the sample (Table 1).

Considering the microhabitats separately, we found the following records. Regarding flowers, the 1,224 individuals sampled are distributed in 29 species, of which $F$. rodeos e $F$. gemina are dominant and comprise $66 \%$ of the individuals, nine are intermediary and 18 are rare (Fig. 4). The species of Frankliniella are generally found on flowers (Mound \& Marullo, 1996). In this study, they prevailed on flowers, but were also registered on shoots and in the leaf litter. However, those sampled in the leaf litter had frequencies below $1 \%$, suggesting their presence there is accidental. On branches, 556 individuals were registered in 26 species. Paraleucothrips minusculus Johansen, 1983, Neohydatothrips flavens (Moulton, 1941), Craniothrips urichi Bagnall, 1915 and Haplothrips sp. are dominant representing $67 \%$ of the sample (Fig. 4). Another seven species are intermediary and 15 are rare. In the grass tussocks we computed 249 individuals in 11 species (Fig. 4). In this microhabitat the dominance of $S$. particula $(83 \%)$ in relation to the others is worth mentioning. Five species had intermediary abundance. With few exceptions, species associated to grasses are almost all exclusive (see below). In the leaf litter 158 individuals were registered in 33 species, of which two are dominant, Adraneothrips alternatus Hood, 1925 and Eschatothrips sp.1 (summing up to $29 \%$ of the individuals), 18 are considered intermediary and 13 are rare (Fig. 4). Although the abundance of thrips was lower, the species richness is similar to that on shoots and flowers. Considering Ananthakrishnan (1993), the leaf litter is a semipermanent habitat, which is heterogeneous and favours high species diversity.

Thus, T1 stands out because of the abundance of $S$. particula in tussocks, T2 because of the



Fig. 1 - Cumulative number of thrips species, obtained from successive sampling from June 1999 to May 2001 in the Parque

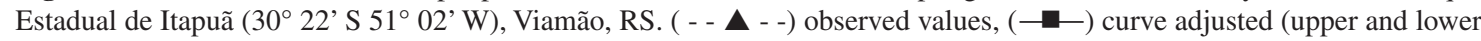
$95 \%$ confidence limits) using the EstimateS version 6.01 software. (axis: $\mathrm{y}=$ cumulative $\mathrm{n}^{\mathrm{o}}$ species, $\mathrm{x}=$ sampling occasion). 

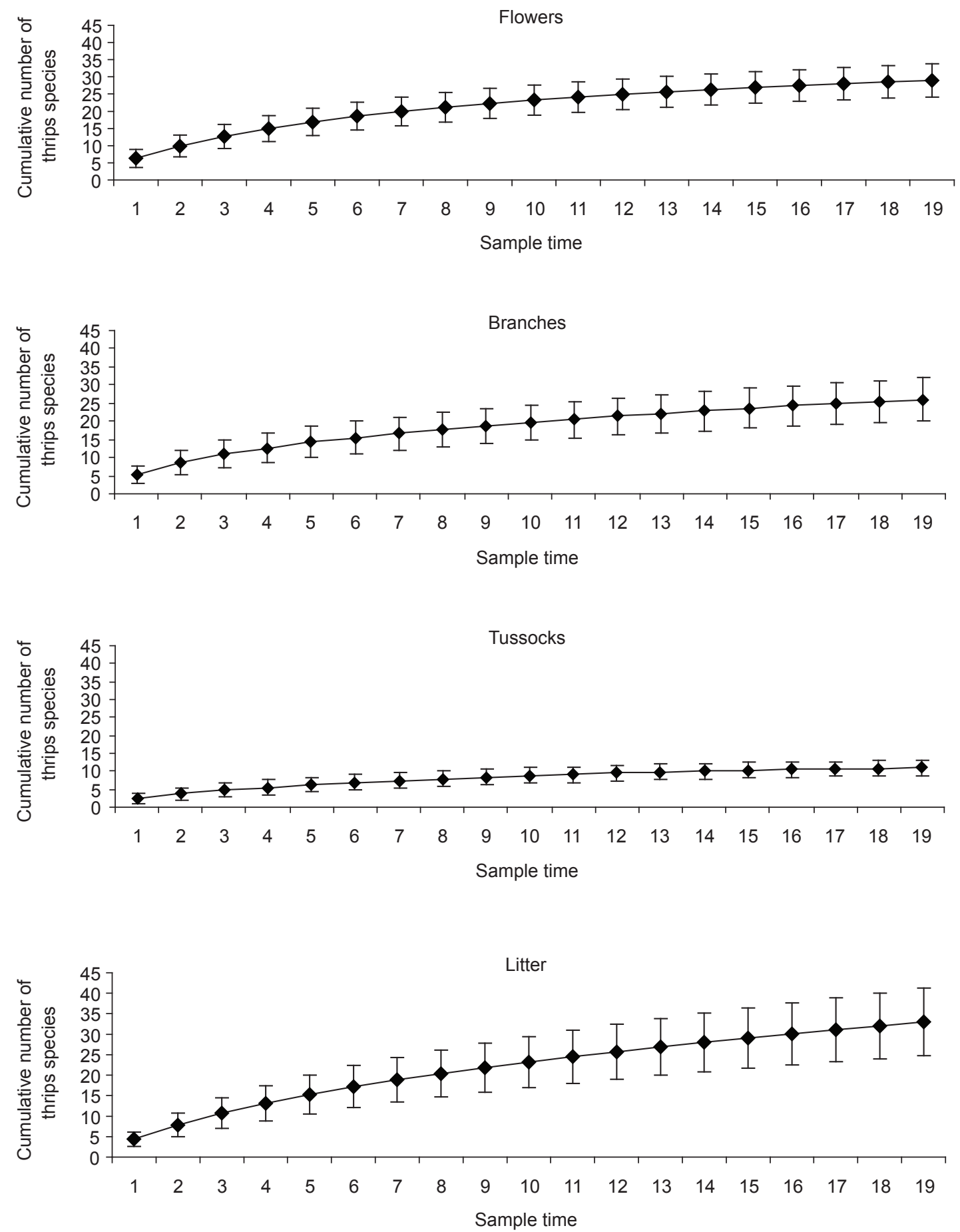

Fig. 2 - Cumulative number of thrips species per microhabitat, obtained from successive sampling from June 1999 to May 2001 in the Parque Estadual de Itapuã (30 22' S 51 ${ }^{\circ}$ 02' W), Viamão, RS. Curve adjusted (upper and lower $95 \%$ confidence limits) using the EstimateS version 6.01 software. (axis: $\mathrm{y}=$ cumulative no. new species, $\mathrm{x}=$ sampling occasion). 




Fig. 3 - Abundance distribution of thrips species (relative frequency per species in order of abundance) found in the Parque Estadual de Itapuã (3022' S 51 02’ W), Viamão, RS. June 1999 to May 2001. (axis: y = relative frequency, x = thrips species).

dominance of the two species of Frankliniella on flowers, $\mathrm{T} 3$ by the high general proportion of $\mathrm{S}$ in relation to $\mathrm{N}$ compared to the other trails and $\mathrm{T} 4$ by the low representation in abundance and richness of the tussock fauna.

The analysis of the occurrence of exclusive species corroborates the differences among environments (Table 2). A high proportion of exclusive species is represented by a single individual overall in the samples (Table 1). In this case, it is difficult to evaluate if a species is a resident with a low populational level or only a "tourist" (Gaston, 1996). However, the high number of species with low abundance and the rising sufficiency curve (Figs. 1 and 2) seems to support the first alternative.

The number of exclusive species in each microhabitat is also worth mentioning. In the leaf litter, 16 exclusive species make up almost $48 \%$ of the individuals. On the branches, eight exclusive species make up $31 \%$ and on flowers, six species make up $21 \%$ of the individuals. In the grass tussocks, out of a total of 11 species, three are exclusive (27\%).

The proportion of exclusive species per microhabitat must be higher. Many species sampled in high abundance in a habitat were also represented in others with only one or a few individuals (for ex. F. rodeos, F. gemina, S. particula, and a good proportion of the dominant species - Table 1). It is reasonable to suppose that, for at least some of these cases, the presence of thrips was occasional (dispersal, sampling). About $20 \%$ of the exclusive species, among trails, were of an intermediary abundance and all the others were rare. Thus it is more difficult to interpret the meaning of exclusivity per trail.

The highest exclusivity occurred on $\mathrm{T} 2$, the trail with the most heterogeneous environment. In general, exclusive species were distributed equally among the microhabitats in the four trails.

Moreover among the trails, the thrips assemblages varied a lot in structure and composition (Fig. 5). In T1, 534 individuals in 32 species were sampled. S. particula, F. gemina and C. urichi were dominant making up $60 \%$ of the sample and seven species had intermediary abundances, comprising $32 \%$ of the individuals. In this trail vegetation has relatively little impact. The presence of $S$. particula is related to the abundance of Homolepis glutinosa (Sw.) Zuloaga \& Soderstr (Poaceae). It is possible that the shade produced by the forest present in this trail has lowered the frequency of flowers and, by association, is related to the less characteristic presence of the genus Frankliniella (see below).

$\mathrm{T} 2$ had the highest abundance and highest species richness - 935 individuals in 42 species were sampled. F. rodeos, F. gemina, and Frankliniella insularis (Franklin, 1908) were dominant on this trail and contributed with $60 \%$ of the individuals. Nine species were intermediary and 30 rare. The trail experienced much anthropic action in the past, though nowadays it is at varied stages of regeneration. The good rate of sunlight, resulting from past perturbations favours the abundance of flowers indirectly to which the species associate. Thus, it has heterogeneous environments and the 
Flowers
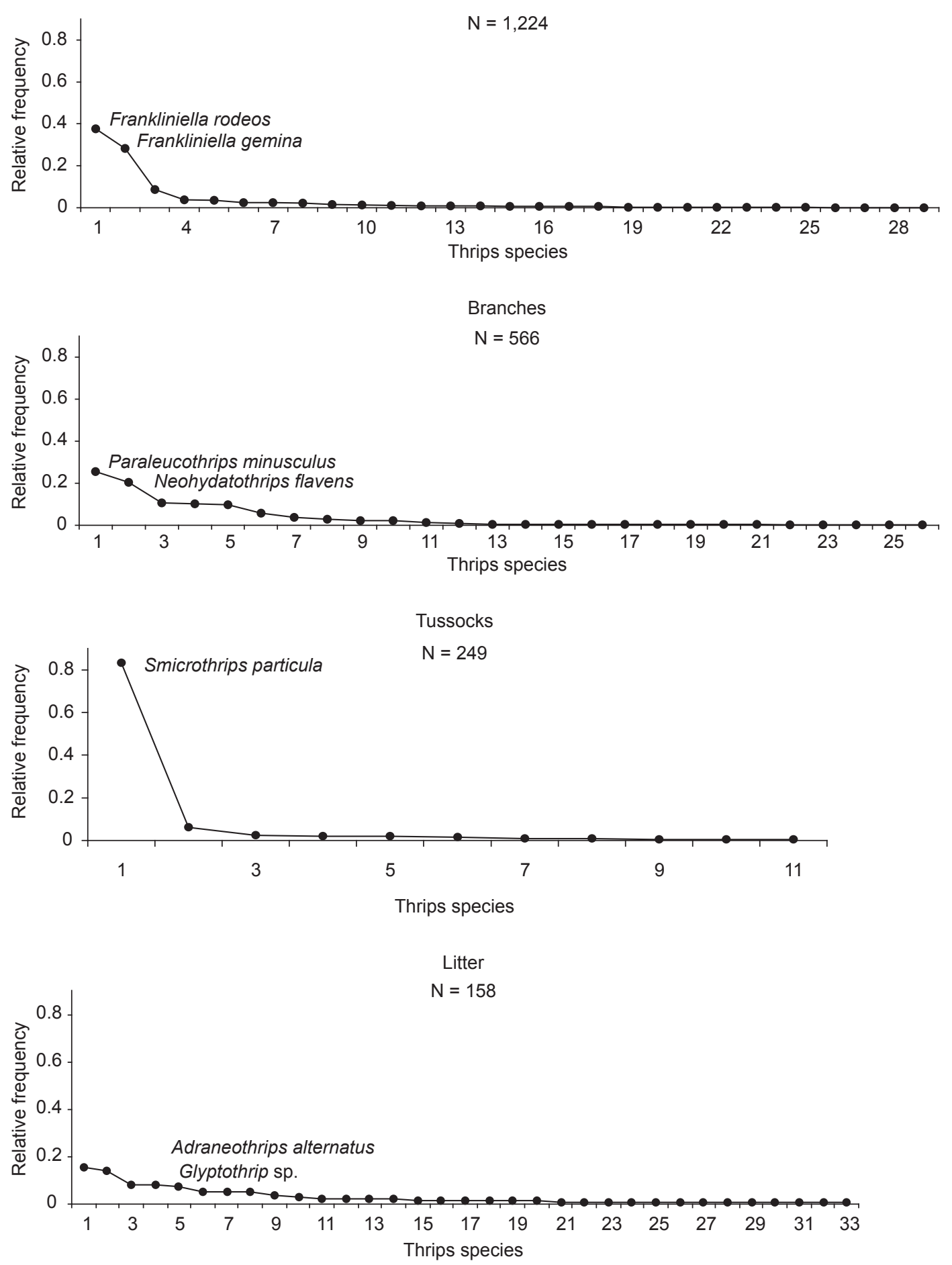

Fig. 4- Abundance distribution of thrips species (relative frequency per species in order of abundance) in four microhabitats (flowers, shoots, grass tussocks and leaf litter), sampled in the Parque Estadual de Itapuã (30²2' S 51 $02^{\circ}$ 'W), Viamão, RS. June 1999 to May 2001. (axis: $y=$ relative frequency, $x=$ thrips species). 
TABLE 2

Number of exclusive thrips species per trail and for all of PEI, listed by microhabitat. Note that exclusive species from one trail, found in a certain microhabitat, are not necessarily exclusive to that microhabitat and thus the values for the whole of the PEI are not the sum of the values for each trail, and the totals for each trail is not necessarily the sum of the values per microhabitat.

\begin{tabular}{|l|c|c|c|c|c|}
\hline Trail/Microhabitat & Flowers & Branches & Tussocks & Litter & Total \\
\hline T1 & 3 & 1 & 2 & 2 & 8 \\
\hline T2 & 3 & 3 & - & 6 & 12 \\
\hline T3 & - & 3 & 1 & 3 & 7 \\
\hline T4 & - & 1 & - & 5 & 6 \\
\hline PEI & 6 & 8 & 3 & 16 & 33 \\
\hline \% total of species & 21 & 31 & 27 & 48 & 45 \\
\hline
\end{tabular}

distinct plant communities which were found favour the diversity of others organisms.

T3 had 243 individuals and 29 species, with P. minusculus, $N$. flavens and F. rodeos the dominant species, with $46 \%$ of the individuals. Another 15 species are intermediary and 11 rare. This trail used to be inhabited by local fishermen and visited by beach goers. Its restinga environment is marked by the presence of dunes, with typically xerophytic vegetation. It has (among other formations) bushy secondary vegetation, where $N$. flavens was collected in abundance. The abundance of $F$. rodeos in $\mathrm{T} 2$ and $\mathrm{T} 3$ could be associated to the presence of Cordia verbenacea DC. (Boraginaceae), Rubiaceae gen. et sp. indet. and Dodonea viscosa L. (Sapindaceae) on both trails.

Considering T4 we registered 485 individuals within 36 species. F. rodeos, N. flavens and F. gemina are dominant (54\% of the individuals), 11 species are intermediary, contributing with $38 \%$ of the individuals and 20 are rare containing only $8 \%$ of the sample (Fig. 5). This trail passes by the intermediary stratum between the low and high forest of the Grota hill. The rupestral vegetation, where the bushy secondary vegetation is markedly present, favours the expressive abundance of $N$. flavens associated to $D$. viscosa.

The faunas, of which are very peculiar in each trail, resulted in a very distinct relationship between species richness $(\mathrm{S})$ and the number of individuals (N) per microhabitat (Fig. 6). As discussed above, particular conditions at each place, the history of use and plant species formations present in each trail combine to determine particular thysanopterofaunas.
The relationship between species richness $(\mathrm{S})$ and the number of individuals $(\mathrm{N})$ on the microhabitats varied enormously among the trails (Fig. 6), which were also diversified having very peculiar faunas.

The analysis of the occurrence or not of exclusive species on the trails indicated that the former were found in all four environments. About $20 \%$ of these exclusive species were intermediary, whilst the others were represented by only one individual considering the overall sample. In the latter case, it is difficult to evaluate whether a species was particular or whether the population level was or used to be low, making its sampling improbable. The highest incidence of exclusive species occurred in $\mathrm{T} 2$.

Many associations, presumably, exist between the diversity of flowers and the diversity of thrips feeding on the plants in general (Mound, 2002). However, probably $50 \%$ of all the species feed only on fungi (Mound \& Palmer, 1983). Among these, there are species belonging to Phlaeothripidae and all Idolothripinae are fungivores, whilst species of Phlaeothripinae have a varied biology. Furthermore, most of the species feed on flowers, particularly grasses (Mound \& Kibby, 1998). In the present work the Phlaeothripinae species were plentiful, especially those in the leaf litter.

The geomorphic and physiognomic-floristic formation of these four trails has a reflection in the diversity and abundance of thysanopteran species sampled in the different environments composing the "Parque Estadual de Itapuã". The marked variation observed in the thysanopterofauna among microhabitats and trails, on the other hand, 


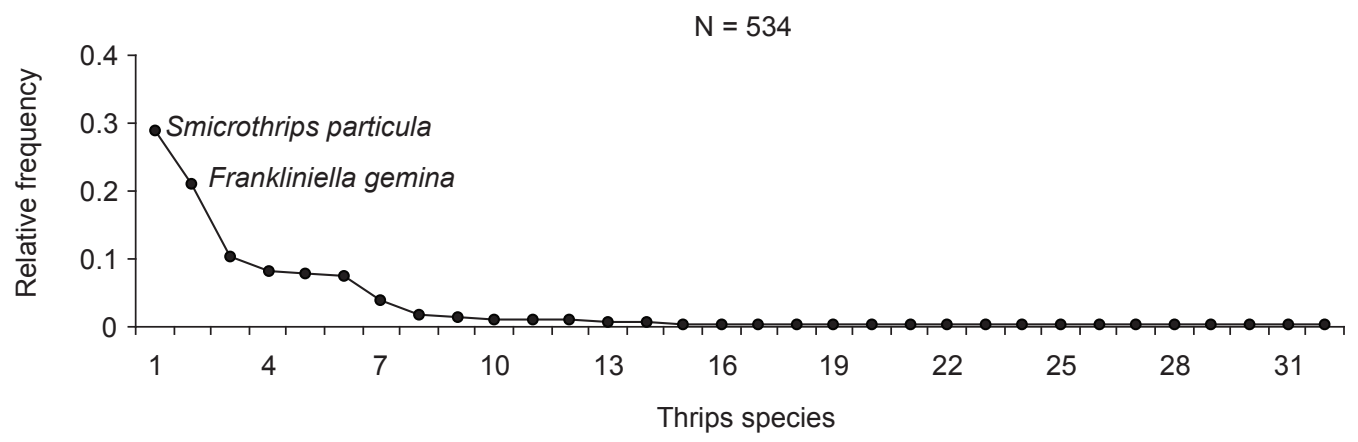

T2
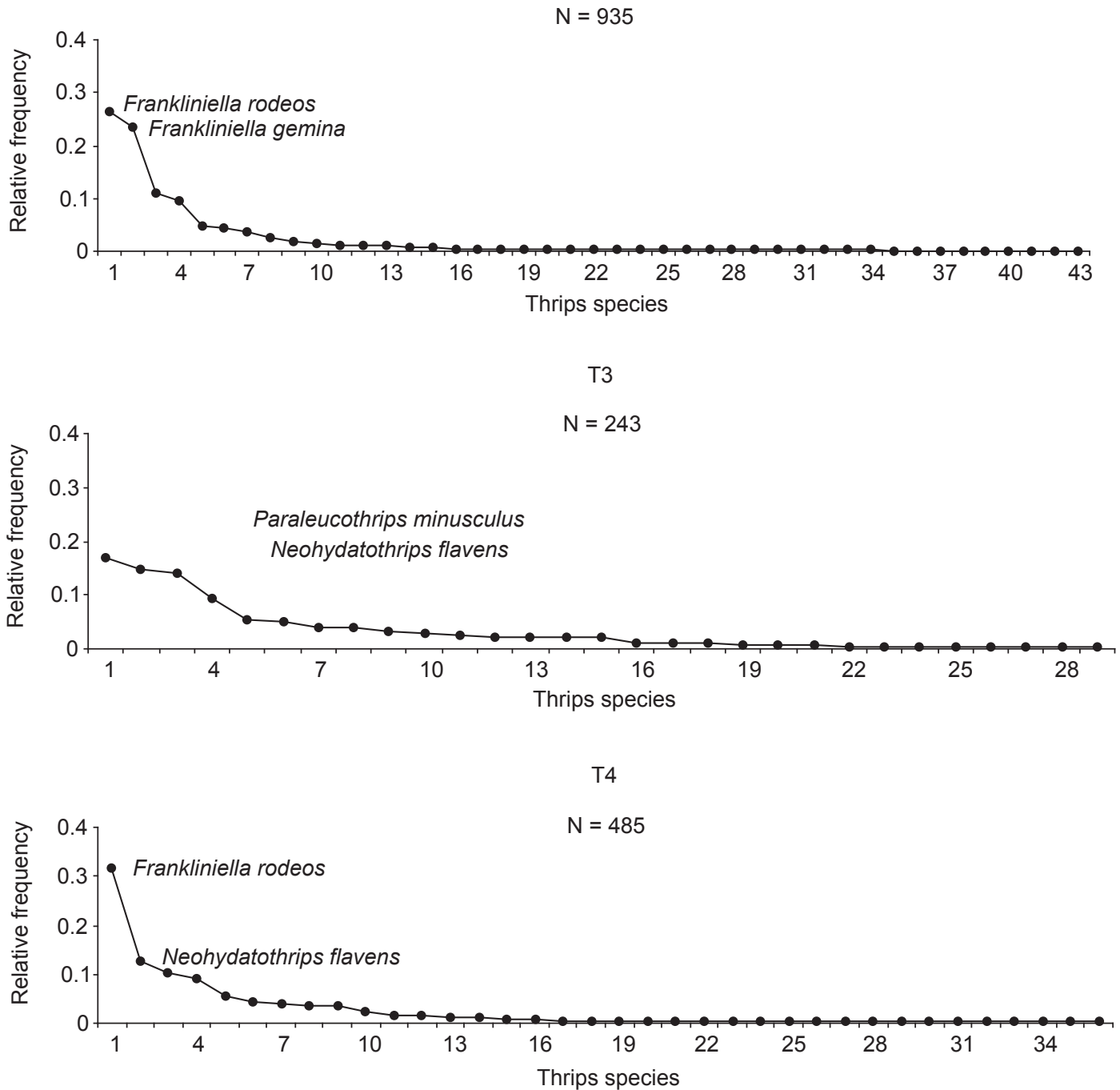

Fig. 5 - Abundance distribution of thrips species (relative frequency per species in order of abundance) in four environments, sampled in the Parque Estadual de Itapuã (30²2' S 51 02’ W), Viamão, RS. June 1999 to May 2001. T1 (Pedreira beach), T2 (Araçá beach), T3 (Lagoinha), T4 (Grota hill). (axis: $y=$ relative frequency, $x=$ thrips species). 


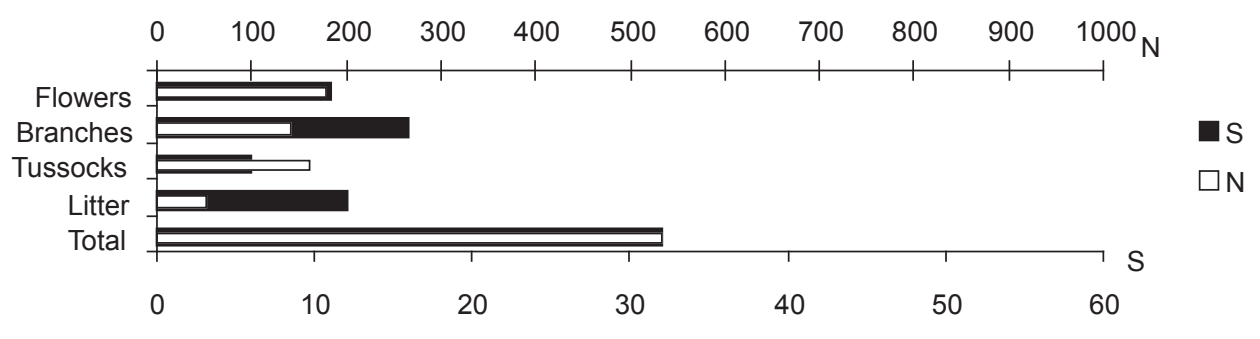

T2

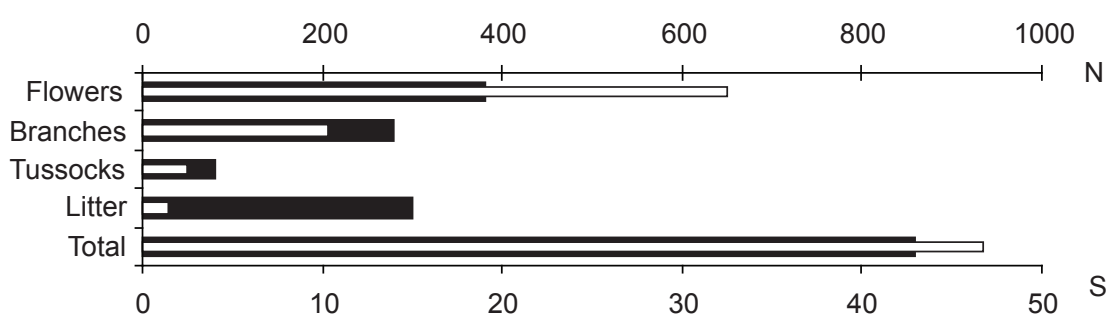

T3
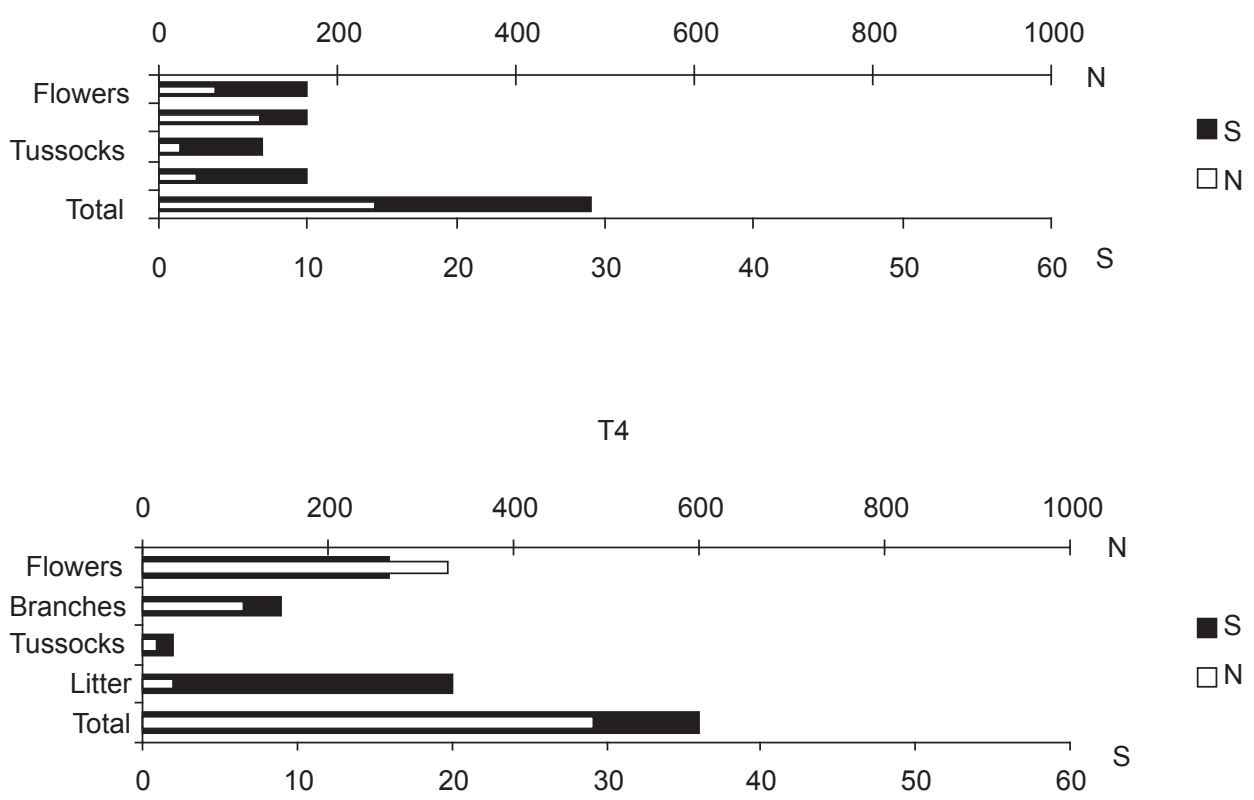

Fig. 6 - Species richness $(\mathrm{S})$ and number of individuals $(\mathrm{N})$ of thysanopterans per microhabitat (flowers, branches, grass tussocks and leaf litter) and per trail (T1 - Pedreira beach, T2 - Araçá beach, T3 - Lagoinha, T4 - Grota hill) in the Parque Estadual de Itapuã (30²2’ S 51²02’ W), Viamão, RS. June 1999 to May 2001. 
highlights the urgency for surveys which include a wide range of environments and also how much we still have to know about insect diversity in general and, particularly, that of the Thysanoptera.

Acknowledgments - To the Conselho Nacional de Desenvolvimento Científico e Tecnológico (CNPq), Brazil for the financial support, Departamento de Florestas e Áreas Preservadas da Secretaria Estadual do Meio Ambiente do Rio Grande do Sul (DEFAP) for the authorization to work in the Parque Estadual de Itapuã and Dr. Laurence A. Mound who observed and identified several species of thrips.

\section{REFERENCES}

ANANTHAKRISHNAN, T. N., 1993, Bionomics of Thrips. Anпи. Rev. Entomol., 38: 71-92.

BRACK, P., SCHÜTZ, R. S. \& SOBRAL, M., 1998, Árvores e arbustos na vegetação natural de Porto Alegre, Rio Grande do Sul, Brasil. Iheringia, Sér. Bot., 51: 139-166.

COLWELL, R. K., 2000, EstimateS: Statistical Estimation of Species Richness and Shared Species from Samples, Versão 6.01 - http://|viceray.eeb.uconn.edu/estimates.

CRONQUIST, A., 1988, The evolution and classification of flowering plants. The New York Botanical Garden, New York, 555p.

DEL-ClARO, K., MARUllO, R., \& MOUND, L. A., 1997, A new Brazilian species of Heterothrips (Insecta: Thysanoptera) co-existing with ants in the flowers of Peixotoa tomentosa (Malpighiaceae), J. Nat. Hist., 31: 1307-1312.

DE SANTIS, L., GALLEGO DE SUREDA, A. \& MERLO, Z., 1980, Estudio Sinoptico de los tisanopteros Argentinos (Insecta). pp. 91-166. In: Obra del centenario de Museo de La Plata. La Plata: Secretaria de Ciência y Tecnologia de La Nación,

DeVRIES, P. J., MURRAY, D. \& LANDE, R., 1997, Species diversity in vertical, horizontal, and temporal dimensions of a fruit-feeding butterfly community in a Ecuadorian rainforest. Biol. J. L. Soc., 62: 343-364.

GASTON, K. J., 1996, Biodiversity: a biology of numbers and difference. Blackwell Science, Oxford 396p.

HOOD, J. D., 1952, Brazilian Thysanoptera III. Proc. Biol. Soc. Wash., 65: 173.

KIRK, W. D. J., 1996, Thrips. In: The Richmond Publishing Co. Ltd (ed.), Naturalist's Handdooks, 25: 70p.

LEWINSOHN, T. M., PRADO, P. K. L. \& ALMEIDA, A. M., 2001, Inventários bióticos centrados em recursos: insetos fitófagos e plantas hospedeiras, pp. 174-189. In: Dias, B. F. S. \& Garay, I. (orgs.) Conservação da biodiversidade em ecossistemas tropicais. Avanços conceituais e revisão de novas metodologias de avaliação e monitoramento, Vozes, Porto Alegre.

LEWIS, T., 1973, Thrips. their biology, ecology, and economic importance, Academic Press, London 349p.

LORENZI, H. \& MOREIRA, H. S., 1999, Plantas ornamentais no Brasil: arbustivas, herbáceas e trepadeiras. 2.ed. rev. and ampl. Instituto Plantarum, Nova Odessa, 1.088p.
MONTEIRO, R. C., MOUND, L. A. \& ZUCCHI, R. A., 1996, Thrips species from three counties of state of São Paulo $A n$. Soc. Entomol. Brasil, 25: 351-353.

MONTEIRO, R. C., MOUND, L. A. \& ZUCCHI, R. A., 1999, Thrips (Thysanoptera) as pests of plants production in Brazil. Revta. Bras. Ent., 43: 163-161.

MONTEIRO, R. C., MOUND, L. A. \& ZUCCHI, R. A., 2001, Espécies de Frankliniella (Thysanoptera: Thripidae) de Importância Agrícola no Brasil, Neotrop. Entomol., 1: 65-72.

MORITZ, G., MORRIS, D. \& MOUND, L. A., 2001, Thrips ID: Pest thrips of the world, Colling, CSIRO publishing, $1 \mathrm{CD}-\mathrm{ROM}$

MOULTON, D., 1948, The genus Frankliniella Karny, with keys for the determination of species (Thysanoptera). Rev. Entomol., 19: 55-114.

MOUND, L. A., 2000, The aquatic thrips Organothrips indicus Bhattti (Thysanoptera: Thripidae) in Queensland, and a new species, O. wrighti, from tropical Australia. Aust. J. Ent., 39: 10-14.

MOUND. L. A., 2002, Thysanoptera biodiversity in the Neotropics. Rev. Biol. Trop., 50: 477-484.

MOUND, L. A. \& O'NEILL, K., 1974, Taxonomy of the Merothripidae, with ecological and phylogenetic considerations (Thysanoptera). J. nat. Hist., 8: 481-509.

MOUND, L. A. \& PALMER, J. M., 1983, The generic and tribal classifation of spore-feeding Thysanoptera (Phlaeothripidae: Idolothripinae), Bull. Britsh Mus. (Natural History) Ent., 46: 1-174.

MOUND, L. A. \& MARULLO, R., 1996, The thrips of Central and South America: an introduction (Insecta: Thysanoptera). (Memoirs on Entomology International), $6^{\circ}$ vol., $487 \mathrm{p}$., Florida.

MOUND, L. A. \& KIBBY, G., 1998, Thysanoptera: an identification guide, 2. ed. Wallingford: CAB, 70p.

PALMER, J. M., MOUND, L. A. \& HEAUME, G. J., 1989, Thysanoptera (Cie Guides to insects of importance to man) $2^{\circ}$ vol., 74p., CAB, Wallingford.

PINENT, S. M. J., ROMANOWSKI, H. P., REDAELLI, L. R. \& MOUND, L. A., 2003, thrips Species (Thysanoptera) Collected at Parque Estadual de Itapuã, Viamão, RS, Brazil, Neotrop. Entomol., 32: 619-623.

RIO GRANDE DO SUL, 1997, Plano de Manejo Parque Estadual de Itapuã. Porto Alegre. Secretaria da Agricultura e Abastecimento. Departamento de Recursos Naturais Renováveis, $158 \mathrm{p}$.

RIPA, R. S., RODRIGUEZ, F. A. \& ESPINOZZA, M. H., 2001, El Trips de California em Nectarinos y Uva de Mesa. La Cruz, Boletín INIA, 53:100p.

SAKIMURA, K. \& O’NEILL, K., 1979, Frankliniella, redefinition of genus and revision of minuta group species (Thysanoptera: Thripidae). Department of Agriculture (Technical Bulletin, 1572) 49p. 
